\title{
Prescribing pattern of drugs in chronic kidney disease patients on hemodialysis at a tertiary care hospital
}

\author{
Narayana Murthy B. V.*, Satyanarayana V.
}

Department of Pharmacology, Rajrajeswari Medical College, Bangalore, Karnataka, India

Received: 31 January 2017

Revised: 03 February 2017

Accepted: 28 February 2017

\section{*Correspondence to:}

Dr. Narayana Murthy B. V., Email:

drnmurthybv@gmail.com

Copyright: (C) the author(s), publisher and licensee Medip Academy. This is an openaccess article distributed under the terms of the Creative Commons Attribution NonCommercial License, which permits unrestricted noncommercial use, distribution, and reproduction in any medium, provided the original work is properly cited.

\begin{abstract}
Background: CKD (chronic kidney disease) is a general term for heterogeneous disorders affecting kidney structure and its function. It is defined as either kidney damage or a decreased glomerular filtration rate of less than 60 $\mathrm{mL} / \mathrm{min} / 1.73 \mathrm{~m}^{2}$ for 3 or more months. Objective of present work is to study the drug utilization pattern in chronic kidney disease patients undergoing hemodialysis.
\end{abstract}

Methods: After taking the clearance and approval from the institutional ethics committee, a cross sectional prospective observational study conducted on chronic kidney disease (CKD) patients undergoing hemodialysis, in the department of nephrology, of Rajarajeshwari Medical College and Hospital, Bangalore. The data was analyzed descriptively.

Results: Study included 52 patients, among them 41 males, 11 were females, with a mean age of 47.6yrs. In our study large number pt were suffering from hypertension (HTN) $88.46 \%$ (46), in them the calcium channel blocker (CCB) $08.48 \%$ (38) was most commonly prescribed anti hypertensive drug. Around $1 / 3$ of pt suffering from diabetes mellitus (DM) 36.53\% (19) most of these patients were treated with them treated with oral hypoglycemic agents (OHA), and less than half of pt treated with insulin01.56\% (07). Other drugs like phosphate binders (calcium carbonate \& acetate) used in $11.16 \%$ (50), aspirin in $08.70 \%$ (39), statins in $10.04 \%$ (45) pt were being most commonly prescribed drugs. Totally 448 drugs were prescribed In 52 pts Ie about 8.61 drugs / prescription, showing poly pharmacy.

Conclusions: Patients undergoing hemodialysis with CKD will be having multiple diseases associated, regular monitoring and counseling regarding these diseases and its complication may reduce the incidence of CKD and the mortality and morbidity associated. The poly pharmacy noted in the study found inevitable because of the multifactorial etiologiesinvolved and needful multiinterventional approach towards it.

Keywords: CKD, Drug utilization, Hemodialysis

\section{INTRODUCTION}

CKD (chronic kidney disease) is a general term for heterogeneous disorders affecting kidney structure and its function. It is defined as either kidney damage or a decreased glomerular filtration rate of less than 60 $\mathrm{mL} / \mathrm{min} / 1.73 \mathrm{~m}^{2}$ for 3 or more months. ${ }^{1-3}$

CKD is a key determinant of poor health outcomes for major Non-communicable diseases (NCDs), and it is a worldwide threat to public health. Estimates of the global burden of the diseases report that kidney and urinary tract diseases contribute 830000 deaths annually making them the 12th highest cause of death (1.4\% of all deaths) and disability-adjusted life years (DALY) in 18867000, i.e. 17 th cause of disability ( $1 \%$ of all DALY). ${ }^{4-7}$

Chronic kidney disease (CKD) is a global threat to health in general and for developing countries in particular because of its increasing incidence, poor outcome, and high cost of treatment. ${ }^{1}$ The treatment in CKD patients includes renal transplantation and dialysis, it will be according to the stage of the disease and these are considered in later stages of CKD. The first step in the 
treatment is to determine the underlying cause and treating it. Initiation of dialysis in advanced CKD along with the management of clinical manifestations due to it. ${ }^{7,8}$ In India, the prevalence of CKD was observed to be $17.2 \%$ with $\sim 6 \%$ have CKD Stage 3 or worse (25) and it is more seen among males compared to females due to stress, alcoholism, hypertension, diabetes mellitus, smoking and cumulative risk factors of chronic vascular disease (CVD). ${ }^{4-9}$ Diabetes mellitus, hypertension, smoking, cardiovascular diseases (CVD), age, chronic use of non-steroidal anti-inflammatory drugs (NSAIDs) and obesity are being the major cause of CKD. ${ }^{10} \mathrm{CKD}$ Patients do not have symptoms until the kidney function is severely impaired and as the disease advances, symptoms like loss of appetite, somnolence, uremia, nausea, vomiting, and confusion may develop. Patients also suffer from hypertension, electrolyte abnormalities like hyperkalemia, hypocalcaemia, hyperphosphatemia, anemia and renal osteodystrophy.

Dialysis (from Greek meaning dissolution, dia means through, lysis means loosening or splitting) is a process for removing waste and excess water from the blood, and is used primarily as an artificial replacement for lost kidney function in people with renal failure. Hemodialysis, also called as an artificial kidney, has already been proven as an adjunct of life-saving therapy in case of acute renal failure and mainly chronic renal failure. ${ }^{11-13}$ Anemia is the next major complication of CKD and patients are treated with erythropoietin or iron supplements. Other drugs given for CKD patients include diuretics and potassium binding resin for hyperkalemia. Calcium salts or phosphate binders to treat hyperphosphatemia, sodium bicarbonate to manage metabolic acidosis, calcium and vitamin D supplementation for renal osteodystrophy and drugs for other co-morbidities. $^{7,8}$

Appropriate drug selection for patients with chronic kidney disease (CKD) is important in order to avoid unwanted drug effects and to ensure optimal patient outcomes. Rational drug prescription is a difficult task in CKD patients. These patients are at higher risk of drugrelated problems since they need complex therapeutic regimens that require frequent monitoring and dosage adjustments. In addition, they usually have other co morbidities. $^{6,14-19}$ Inappropriate use of medications can increase adverse drug effects, which can be reflected by excessive length of hospital stays, excessive health care utilization, and costs. ${ }^{14,20-22}$ As CKD progresses, the drugs that are given for these patients also increases and the prevalence of drug related problems also increases. Patients with CKD and on maintenance hemodialysis are prescribed with an average of 12 medications per patient and are at a higher risk of developing drug related problems thus leading to untoward effects. ${ }^{7,8}$ Dialysis patients require special consideration regarding the drugs used because of their altered pharmacokinetic and pharmacodynamics profiles and they have increased potential for adverse reactions.

\section{METHODS}

This is a prospective, observational study conducted on CKD patients undergoing hemodialysis (includes both the inpatient and outpatients), in the Department of Nephrology, of Rajarajeshwari Medical College and hospital, Bangalore. After taking the clearance and approval from institutional ethical committee the study will be undertaken for a period of 6 months. Relevant data will be collected on a Proforma which is designed according to the protocol of the study which includes mainly about the drugs.

- Dose, dosage, duration, timing of administration, route of administration of the prescribed drugs.

- Change of drugs during the treatment to any reason will also be noted.

- Investigations done and its reports, which are relevant to the study will be noted.

\section{Inclusion criteria}

All CKD patients undergoing hemodialysis aged above 18 years.

\section{Exclusion criteria}

- Patients on hemodialysis for ARF (acute renal failure).

- Patients not willing to give consent.

\section{Statistical analysis}

The data collected will be analyzed statistically using descriptive statistics. Wherever necessary, the results depicted in the form of percentages and graphs.

\section{RESULTS}

The study involved 52 CKD patients undergoing hemodialysis. Among these $79 \%$ (41) male and $21 \%$ (11) was female with a mean age of 47.6 years (Figure 1, Table 1).

Table 1: Prescription analysis.

\begin{tabular}{|l|l|}
\hline Details of prescription & Number \\
\hline Male & 41 \\
\hline Female & 11 \\
\hline Mean average age & 47.6 years \\
\hline Prescription analyzed & 52 \\
\hline Total no drugs prescribed & 448 \\
\hline No FDC observed & 40 \\
\hline No of drugs prescribed by generic names & 0 \\
\hline $\begin{array}{l}\text { Number of drugs from WHO essential } \\
\text { drug list }\end{array}$ & 178 \\
\hline
\end{tabular}

Totally 448 drugs, as 8.61 drugs/ prescription prescribed in the study showing poly pharmacy, and in these drugs 
about 178 (39.75\%) drugs only prescribed from WHO essential drug list and 40 FDC were prescribed in our study (Table 1).

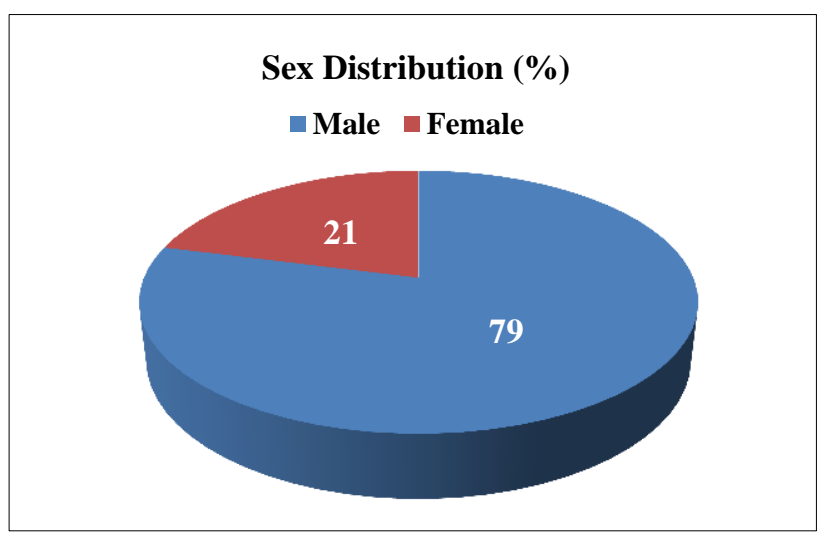

Figure 1: Sex ratio in CKD patients.

The risk and co morbidities associated with these pts was hypertension $88.46 \%$ (46), smoking $75 \%$ (39), alcoholics $53.84 \%$ (28), diabetes mellitus $36.53 \%$ (19), anemia $36.53 \%$ (19), coronary artery disease $36.53 \%$ (19), and thyroid disorder $5.76 \%$ (03) (Table 2).

Table 2: Risk and co morbidities associated with CKD.

\begin{tabular}{|ll|}
\hline Risk and co-morbidities & No and \% of patients \\
\hline HTN & $46(88.46)$ \\
\hline Smoking & $39(75.00)$ \\
\hline Alcohol & $28(53.84)$ \\
\hline DM & $19(36.53)$ \\
\hline Anemia & $19(36.53)$ \\
\hline CAD & $19(36.53)$ \\
\hline Thyroid & $03(05.76)$ \\
\hline
\end{tabular}

In diabetes mellitus $17(89 \%)$ patients were suffering from type 2 and $2(11 \%)$ of type 1 (Figure 2). The drugs prescribed to these pts with above co morbidities and risk factors are summarized in (Table 3 ).

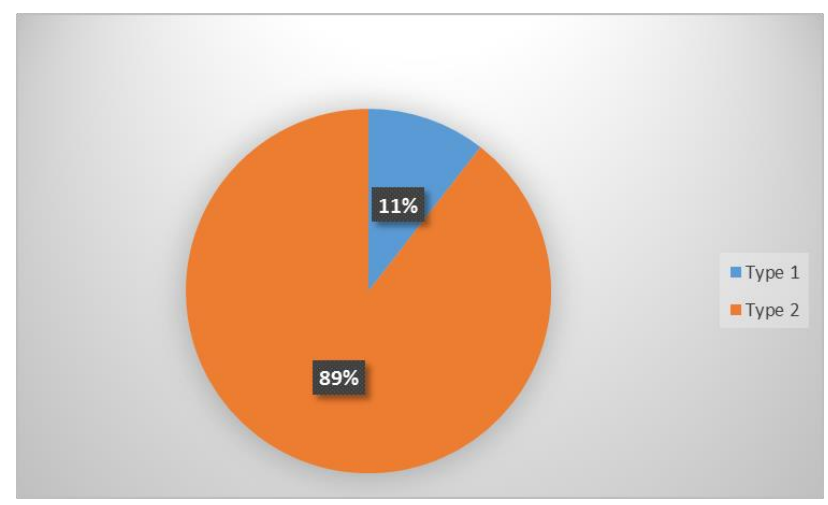

Figure 2: Percentage of patients in type 1 and 2 DM.

Cardiovascular drugs were most commonly used drugs $42 \%$, followed by hematopoietic $15 \%$, phosphate binders
$11 \%$, gastro intestinal tract $10.9 \%$, multivitamins $10.9 \%$, DM 09\%.

Table 3: Percentage of drug classes used in different systems.

\begin{tabular}{|lll|}
\hline Drug Class & No of drugs & \% of drugs \\
\hline CVS & 189 & 42.18 \\
\hline DM & 40 & 08.92 \\
\hline GIT & 49 & 10.93 \\
\hline Phosphate binders & 50 & 11.16 \\
\hline Hematopoietic & 68 & 15.17 \\
\hline Multi vitamins & 49 & 10.93 \\
\hline Thyroid & 03 & 00.66 \\
\hline Total & 448 & \\
\hline
\end{tabular}

The five most commonly prescribed drugs are statins $10.04 \%$, diuretics $9.15 \%$, aspirin $8.70 \%$, calcium channel blockers $8.48 \%$, OHA $7.36 \%$ (Table 4).

Table 4: Percentage of different drugs used.

\begin{tabular}{|lll|}
\hline Drugs & Numbers & Percentage \\
\hline CVS & 189 & 42.18 \\
\hline CCB & 38 & 08.48 \\
\hline ACE & 20 & 04.46 \\
\hline ARB & 12 & 02.67 \\
\hline Alpha 2 agonists & 25 & 05.58 \\
\hline Beta blockers & 04 & 01.33 \\
\hline Diuretic & 41 & 09.15 \\
\hline Others & 49 & 10.93 \\
\hline GIT & 49 & 10.93 \\
\hline H2 blockers & 09 & 02.00 \\
\hline PPI & 40 & 08.92 \\
\hline DM & 40 & 08.92 \\
\hline OHAs & 33 & 07.36 \\
\hline Insulin & 07 & 01.56 \\
\hline Phosphate binders & 50 & 11.16 \\
\hline Calcium carbonate & 28 & 06.25 \\
\hline Calcium acetate & 22 & 04.91 \\
\hline Multi vitamins & 49 & 10.93 \\
\hline Hematopoietic & 68 & 15.17 \\
\hline Thyroid & 03 & 00.66 \\
\hline
\end{tabular}

\section{DISCUSSION}

Modernization and changing life style in developing countries like India has enormously increased the statistical figures of alcohol use, smoking and also noncommunicable diseases like diabetes mellitus, hypertension, obesity, cardiovascular disease, and its complications like, myocardial infarction, stroke, retinopathy, chronic kidney disease. These are due to lack of awareness about the disease and its management. In present study on CKD points undergoing hemodialysis, reveled majority of patients were males $78.84 \%$ (41) showing the similar gender distribution in earlier studies 
done by Bajait et al and Chakraboty et al, most of these patients aged between 41-60 years with a mean age of 48 yrs and the lowest was $24 \mathrm{yr}$ old patient with type $1 \mathrm{DM}$.

Several risk factors and comorbidities were found to be associated among these CKD pts, which is similar to the studies conducted earlier, and they are.

- Hypertension $88.46 \%$ (46)

- $\quad$ Smoking 75\% (39)

- Alcoholics 53.84\% (28)

- Diabetes mellitus $36.53 \%$ (19)

- Anemia 36.53\% (19)

- Coronary artery disease $36.53 \%$ (19) and

- $\quad$ Thyroid disorder $5.76 \%(03)$

In the present study 448 drugs are prescribed, no drugs were prescribed by generic names, showing that prescribing by brand name is the norm, which needs to be discouraged. Encouraging writing of prescription by generic names is always recommended by various national and international bodies to promote rational use of medicines. $40 \%$ of the prescribed drugs were from the WHO essential medicines list. Showing similarity with the study done by Bajait et al.

Making the other drugs which are used in the treatment of these pts as freely available in public health facilities is advised because of the increasing incidence and high cost involved in the treatment of the disease. The average number of drugs prescribed for these pts is 8.61 drugs / prescription, showing the polypharmacy. Patients undergoing hemodialysis will have many risk, co morbidities and complications, like DM, HTN, CVD, anemia, salt and water retention, electrolyte imbalance, phosphate retention, secondary hyper para-thyroidism. The treatment of all these medical problems includes fluid restriction, multiple dietary restrictions, and drugs like phosphate binders, vitamin D preparations, antihypertensive, hypo-glycemics, diuretics, vitamins, iron supplements, and a variety of other medications. Considering all these heterogeneous disorders affecting these patients the poly pharmacy noted in the study is acceptable. Many of the studies done earlier shows using of more than 9 drugs Bajait, et al and Chakraboty et al, and even upto 12 drug. ${ }^{23}$

The most commonly used medications in this study were based on these co morbidities and complications involved in treating these patients. Hypertension is the most commonly associated co morbidity seen with these patients. $^{24}$ Diuretics were the most commonly used drug in these patients next to this were CCB. Among the drugs used to treat HTN were CCB's (amlodipine, nifedipine) $08.48 \% \%$, and ACE $4.46 \%$ / ARB's $2.67 \%$ (enalapril and telmesartan). Monotherapy is rarely seen among these, most of the anti HTN drugs were prescribed with diuretics, diuretics prescribed in $09.15 \%$ of pts, like thiazide diuretics were used more in our study which is combined with ACE/ARB's (FDC). Because of its renal protective, reduction of sympathetic over activity and reduction of complication like edema and pulmonary edema.

In present study, Diabetes was the 2nd common co morbidity associated in $36.53 \%$ (19) pts, among these most of them were suffering from type $2 \mathrm{DM}$ and only $3.84 \%$ (2) were of type among anti-diabetic medications OHAs have been used in majority of patients and also insulin in few pts $1.56 \%$ (7). Among the hypoglycemic agents used were more of glibenclamide, glimepiride, glipizide, acarbose and metformin (FDC), use of metformin is very less when compared to other OHA because as it may cause lactic acidosis in renal failure patients. In diabetes also FDC and poly therapy was seen using at least two OHA's together and using inj Insulin and OHA's together but these were seen more in treating HTN pts. Anemia is the most common complication seen among these patients due to reduced erythropoietin secretion. In our study, also 36\% (19) pts had anemia and were treated with hematinic and vitamin supplements. As hematinic, iron supplements $15 \%$ (like livogen, folic acid, iron sucrose) and multivitamins in $11 \%$, were prescribed among vitamins methyl cobalamine used more in the diabetics. In present study calcium carbonate and calcium acetate are used as phosphate binders (11.9\%) and also as calcium supplement as it is affordable because of its low cost by many patients when compared to sevelamer hydrochloride and lanthanum carbonate, which is also similar to the other studies done by Bajait, et al and Chakraboty et al.

Heart burns, nausea and belching will be seen in these patients because of using many drugs,stress and also poor food intake. Proton pump inhibitors like esomeprazole, pantoprazole was used in most of the pts to reduce these symptoms than $\mathrm{H} 2$ blockers, even though the cost for these drugs are high when compared to $\mathrm{H} 2$ blockers. Similar results were also noted in studies done by Bajait et al and Chakraboty et al. ${ }^{17}$

\section{CONCLUSION}

Inclusion of new drugs like ARBs, sevelamer hydrochloride, lanthanum carbonate in the treatment of these pts certainly reduces the mortality and morbidity, but these drugs are costly when compared to old ones so there by increases the treatment cost because already pt are using many drugs and also need them to be consumed for longer periods. We think counselling on needful life style modification and good health education about the disease, management, its complications in the early stage of the disease, certainly reduces the incidence of these diseases and also reduces the cost, mortality and morbidity due to these.

Funding: No funding sources Conflict of interest: None declared

Ethical approval: The study was approved by the Institutional Ethics Committee 


\section{REFERENCES}

1. Levey AS, Coresh J. Chronic kidney disease. Lancet. 2012;379:165-80.

2. National Kidney Foundation. KDOQI clinical practice guidelines for chronic kidney disease: Evaluation, classification, and stratification. Am J Kidney Dis. 2002;39:S1-266.

3. Bajait CS, Pimpalkhute SA, Sontakke SD, Jaiswal KM, Dawri AV. Prescribing pattern of medicines in chronic kidney disease with emphasis on phosphate binders. Indian J Pharmacol. 2014;46:35-9.

4. Couser WG, Remuzzi G, Mendis S, Tonelli M. The contribution of chronic kidney disease to the global burden of major noncommunicable diseases. Kidney Int. 2011;80:1258-70.

5. Fogarty International Centre. Global Burden of Disease for the Year 2001. By World Bank Region for Use in Disease Control. Priorities in Developing Countries, $2^{\text {nd }}$ Ed, 2004. Available from: http://www.pic.nih.gov/dcpp/gbd.html.

6. Perico N, Remuzzi1 G. Chronic kidney disease: a research and public health priority. Nephrol Dial Transplant. 2012;27:iii19-26.

7. Veerappan I, Abraham G. Chronic kidney disease: current status, challenges and management in India. Available from: http://www.apiindia.org/medicine_update_2013/chap 130.pdf

8. Jafar TH, Stark PC, Schmid CH. Progression of chronic kidney disease: the role of blood pressure control, proteinuria, and angiotensin-converting enzyme inhibition: a patient-level meta-analysis. Ann Intern Med. 2003;139:244.

9. Rani NV, Thomas R, Rohini E, Soundararajan P, Kannan G, Thennarasu P. A study on drug related problems in chronic kidney disease patients of a tertiary care teaching hospital in South India. World J Pharmaceu Res. 2014;3:1403-17.

10. CO Alebiosu, OO Ayodele, A Abbas. Chronic Renal Failure at the Olabisi Onabanjo University Teaching Hospital, Sagamu, Nigeria. Afr Health Sci. 2006;6(3):132-8.

11. Anding K, Gross P, Rost JM. The influence of uraemia and haemodialysis on neutrophil phagocytosis and antimicrobial killing. Nephrol Dial Transplant. 2003;18:2067-73.

12. Jaiswal S, Das R, Sharma S, Paudel P, Lamichhane SR. Bacteriological study of urinary tract infection in male patients undergoing dialysis due to chronic kidney disease in tertiary care hospitals in Nepal. J Life Sci. 2013;3(2):2249-8656.

13. Chaudhary A, Stone WJ, Breyer JA. Occurrence of pyuria and patients bacteriuria in asymptomatic hemodialysis. Am J Kidney Dis. 1993;21:180-3.

14. RF"unfst"uck, Ott U, Naber KG. The interaction of urinary tract infection and renal insufficiency. International Journal of Antimicrobial Agents. 2006;72-77.

15. Classen DC, Pestotnik SL, Evans RS, Lloyd JF, Burke JP. Adverse drug events in hospitalized patients: excessive length of stay, extra cost, and attributable mortality. JAMA. 1997;227:301-6.

16. Long CL, Raebel MA, Price DW, Magid DJ. Compliance with dosing guidelines in patients with chronic kidney disease. Ann Pharmacother. 2004;38:853-8.

17. Kappel J, Calissi P. Safe drug prescribing for patients with renal insufficiency. Can Med Assoc J. 2002;166:473-7.

18. Gabardi S, Abramson S. Drug dosing in chronic kidney disease. Med Clin North Am. 2005;89:649. 87.

19. Manley HJ, Cannella CA, Bailie GR, Pater WL. Medication-related problems in ambulatory hemodialysis patients: A pooled analysis. Am J Kidney Dis. 2005;46:669-80.

20. Al-Ramahi R. Medication prescribing patterns among chronic kidney disease patients in a hospital in Malaysia. Saudi J Kidney Dis Transpl. 2012;23(2):403-8.

21. Bonk ME, Krown H, Matuszewski K, Oinonen M. Potentially inappropriate medications in hospitalized senior patients. Am J Health Syst Pharm. 2006;63:1161-5.

22. Fattinger K, Roos M, Verge'res P. Epidemiology of drug exposure and adverse drug reactions in two Swiss departments of internal medicine. Br J Clin Pharmacol. 2000;49:158-67.

23. Manley HJ, Garvin CG, Drayer DK, Reid GM, Bender WL, Neufeld TK et al. Medication prescription pattern in ambulatory hemodialysis patients: Comparison of USRDS to a large not for profit dialysis provider. Nephrol Dial Transplant. 2004;19:1842-8.

24. Singh AK, Farag YM, Mittal BV, Subramanian KK, Reddy SR, Acharya VN et al. Epidemiology and risk factors of chronic kidney disease in India-results from the SEEK (screening and early evaluation of kidney disease) study. BMC Nephrology. 2013,14:114.

Cite this article as: Narayana Murthy BV, Satyanarayana V. Prescribing pattern of drugs in chronic kidney disease patients on hemodialysis at a tertiary care hospital. Int $\mathrm{J}$ Basic Clin Pharmacol 2017;6:928-32. 